\title{
Chronic Delta-9-tetrahydrocannabinol Induces Monoamine Release but Not Conditioned Place Preference
}

\author{
Mohamad Aris Mohd Moklas*,1, Zulkhairi Amom², Mohamad Taufik Hidayat Baharuldin ${ }^{1}$, \\ Che Norma Mat Taib ${ }^{1}$, Fatin Nadzirah Zakaria ${ }^{1}$, Muhammad Khairulasraf Muhammad Yusof ${ }^{1}$, \\ Muhammad Farhan Adzhar ${ }^{1}$ Sharida Fakurazi ${ }^{1}$ and Rusliza Basir ${ }^{1}$
}

\author{
${ }^{1}$ Department of Human Anatomy Faculty of Medicine and Health Sciences Universiti Putra Malaysia 43400 Serdang, \\ Selangor Malaysia \\ ${ }^{2}$ Department of Basic Science Faculty of Health Sciences Universiti Teknologi MARA 42300 Puncak Alam, Selangor \\ Malaysia
}

\begin{abstract}
THC is the primary psychoactive compound of Cannabis sativa and while the human abuse potential of THC is well recognised, knowledge of its reinforcing and dependence-producing effects is limited. In addition to euphoric properties, cannabinoids are known to attenuate learning and memory in both humans and animals but the underlying mechanism is unclear. The present study investigated the effects of chronic THC treatment on CB1 receptor-mediated reward. In addition, DA and 5HT and their metabolites DOPAC, HVA and 5HIAA were measured in the striatum and hippocampus. Chronic THC (0.5, 2.0 and $5.0 \mathrm{mg} / \mathrm{Kg}$ ip, 21 days) had no significant effect on conditioned place preference while DA content of the striatum was increased by chronic THC in a dose-dependant manner.
\end{abstract}

Keywords: THC, reward, CPP.

\section{INTRODUCTION}

Delta-9-Tetrahydrocannabinol (THC) is the primary psychoactive compound of Cannabis sativa, one of the oldest drugs of abuse and a widely consumed illicit drug. Even though the abuse properties of THC in humans are established, knowledge of the reinforcing and dependenceproducing effects of THC are still limited. The evaluation of the rewarding properties of drugs has become prominent in behavioural pharmacology where the conditioned place preference (CPP) paradigm is one of the most commonly used experimental protocols for assessing the rewarding properties of drugs. The rewarding properties of THC in commonly used rodent models remain controversial [1]. The interpretations of results obtained with conditioned place preference (CPP) protocols are limited due to the frequency of false positives and differences in methodological details between laboratories [2]. Studies have shown that almost all drugs of abuse are able to induce reward, producing a positive place preference over a limited range of doses [3, 4]. In contrast the effects of cannabinoid agonists in CPP have not always been consistent between studies, and are reported to either suppress or induce place preference. Positive place preference is dependent on numerous factors, such as the dose used, timing of the injections, and modifications to the protocol procedure. Only a limited range of cannabinoid doses have, however, been used and leading to difficulties in inter

\footnotetext{
*Address correspondence to this author at the Department of Human Anatomy, Faculty of Medicine and Health Sciences Universiti Putra Malaysia, 43400 Serdang, Selangor, Malaysia; Tel: +60389472783

Fax:+60389422341; E-mail: aris@medic.upm.edu.my
}

pretation and comparison between studies [5-7]. Drugs of abuse produce their reinforcing effects through actions in the limbic component of the basal ganglia. This circuit of nuclei is responsible for the influence of motivational, emotional, contextual and information on behaviour [8]. The mesolimbic pathway is one of the neural pathways in the brain that link the ventral tegmentum in the midbrain to the nucleus accumbens in the limbic system. It is one of the four major pathways where the neurotransmitter dopamine is found. This pathway is part of a brain reward circuit that has been thought to play an important role in mediating reinforcing or rewarding effects of drug abuse [9]. This hypothesis suggests that a positive reinforcing effect of drugs would be accounted for by an activation of dopamine transmission. Dopamine is commonly associated with the pleasure system of the brain, providing feelings of enjoyment and reinforcement to motivate a person certain activities. Dopamine is released particularly in areas such as the nucleus accumbens and striatum by naturally rewarding such as food, sex and use of certain drugs. The reinforcing properties of THC may be mediated by its action on the mesolimbic dopamine system, which projects from the VTA to the nucleus accumbens.

\section{MATERIALS AND METHODS}

\section{Subjects}

Male Sprague Dawley rats ( $\mathrm{n}=8$ per group) weighing 250-300 g were housed four per cage and acclimatised to the laboratory conditions for one week before the experiment. Rats were kept in a temperature-regulated $\left(22 \pm 1^{\circ} \mathrm{C}\right)$ room and artificial lighting was provided from 0700 hours to 1900 hours. Food and water were available ad libitum and each 
animal was handled daily through the first week. Experimental testing began seven days after the acclimatisation period and was performed during the light cycle.

\section{Drug Administration}

THC (Sigma UK, 0.5, 2.0 and $5.0 \mathrm{mg} / \mathrm{kg}$ i.p) or vehicle $(1.0 \mathrm{ml} / \mathrm{kg}$ i.p, Cremophor/ethanol/saline, 1:1:18) were administered every 48 hours for 21 days before the start of the conditioned place preference protocol.

\section{Conditioned Place Preference (CPP)}

The CPP apparatus has been described elsewhere [4]. The apparatus consisted of two main square conditioning Perspex compartments $(30 \times 30 \times 40 \mathrm{~cm})$ separated by a guillotine door. Each compartment had different visual cues in the form of black and white or granite wall patterns. Both compartments had the same smooth flooring texture. Experimental design consisted of three phases. During phase I (pre-test phase) animals were placed between the two compartments without the guillotine door and were allowed to explore either side for 10 minutes. Using video tracking system, the amount of time spent in each compartment was calculated. Any rats that significantly displayed a preference for a particular compartment were withdrawn from the experiment. Rats were placed in treatment or control groups using an unbiased design. Each animal was assigned to a randomly chosen compartment and treatment order. Phase II (conditioning phase) consisted of three pairings with drug injection alternated with three pairings with vehicle injection. Control rats received vehicle only for six consecutive days. In the conditioning phase each drug treated rat received either drug or vehicle on alternative days and 10 minutes later was placed for 15 minutes in the drug-paired or vehicle-paired compartment with the guillotine door in position. Phase III (test session) was 48 hours after the last injection, to allow clearance of the drug, and neither drug nor vehicle were injected before placing the rat in the middle of the box without the guillotine door and the time in either compartment measured as in phase I. Data were expressed as differences (mean \pm S.E.M) and percentage changes in the time spent in the paired-compartment during Phase I and III. A one-way ANOVA followed by a post-hoc Tukey's multiple comparison test were used to compare differences between groups.

\section{Measurement of Monoamine Levels in Brain Regions}

Immediately after finishing the CPP test, the rats were killed and their brains quickly removed and dissected on ice. The striatum, nucleus accumbens and prefrontal cortex were removed, weighed and then stored at $-80^{\circ} \mathrm{C}$ prior to the analysis of dopamine (DA), 3,4-dihydroxyphenylacetic acid (DOPAC), 5-hydroxytryptamine (5-HT) and 5-hydroxyindoleacteic acid (5-HIAA) using HPLC with electrochemical detection (HPLC + ECD). Tissues were sonicated in ice-cold $0.2 \mathrm{M}$ perchloric acid (PCA) ( $1 \mathrm{ml})$ containing $0.1 \% \mathrm{w} / \mathrm{v}$ sodium metabisulfite (SMBS), $0.01 \% \mathrm{w} / \mathrm{v}$ EDTA and $0.1 \%$ $\mathrm{W} / \mathrm{V}$ cysteine in Eppendorfs and then centrifuged for 20 mins (14000 rpm) at $4^{\circ} \mathrm{C}$ and the supernatants removed and stored for further analysis. The samples were separated by a HPLC and quantified using electrochemical detection. The system consisted of a Phenomenex Sphereclone (4.6 X
$150 \mathrm{~mm}, 5 \mu \mathrm{M}$ ) column with Phenomenex $\mathrm{C}_{18} 4 \mathrm{~mm}$ Security Guard Column and an HPLC Technology RR/066L solvent pump. The mobile phase consisted of $0.05 \mathrm{M} \mathrm{KH}_{2} \mathrm{PO}, 0.1$ mM EDTA, $0.16 \mathrm{mM}$ OSS, $16 \% \mathrm{MeOH}$ and $\mathrm{PH} 3.0$ with $\mathrm{H}_{3} \mathrm{PO}_{4}$ using a flow rate of $0.6 \mathrm{ml} / \mathrm{min}$. The detector system was an Antec Controller CU-04AZ with VT03 glassy carbon working electrode set at $+0.75 \mathrm{~V}$. The retention times for DA, DOPAC, 5-HT and 5HIAA were 4.7, 7.6, 9.5 and 11.9 mins respectively. The amine values were analysed using one-way ANOVA with post hoc comparisons when appropriate (Tukey's test) for multiple comparisons.

\section{RESULTS}

\section{Conditioned Place Preference}

During the preconditioning phase, rats showed no significant preferences for either of the two compartments. The mean values \pm SEM of the amount of time spent in the black and white or granite patterned compartments during preconditioning sessions were $316.4 \pm 16.3 \mathrm{~s}$ and $283.6 \pm 16.3 \mathrm{~s}$ respectively. In the conditioned phase III trial, vehicle treatment had no effect on either place preference or place aversion. Similarly, THC also induced no significant place preference or aversion at any of the doses $(0.5,2.0 \& 5.0 \mathrm{mg} / \mathrm{kg}$ on alternate days for 21 days) (Fig. 1).

\section{Measurement of Monoamine Levels}

The results from the measurement of DA and its major metabolites in the striatum, prefrontal cortex and nucleus accumbens are shown in Fig. (2). In the striatum, THC $(0.5$ $\mathrm{mg} / \mathrm{kg})$ significantly increased DOPAC levels $(\mathrm{p}<0.05)$ without altering levels of DA and HVA. In contrast, THC $(2.0 \mathrm{mg} / \mathrm{kg})$ elevated levels of DA $(\mathrm{p}<0.05)$ but not DOPAC and HVA. At the highest dose $(5.0 \mathrm{mg} / \mathrm{kg})$ levels of DA $(\mathrm{p}<0.001)$, DOPAC $(\mathrm{p}<0.05)$ and HVA $(\mathrm{P}<0.01)$ were all

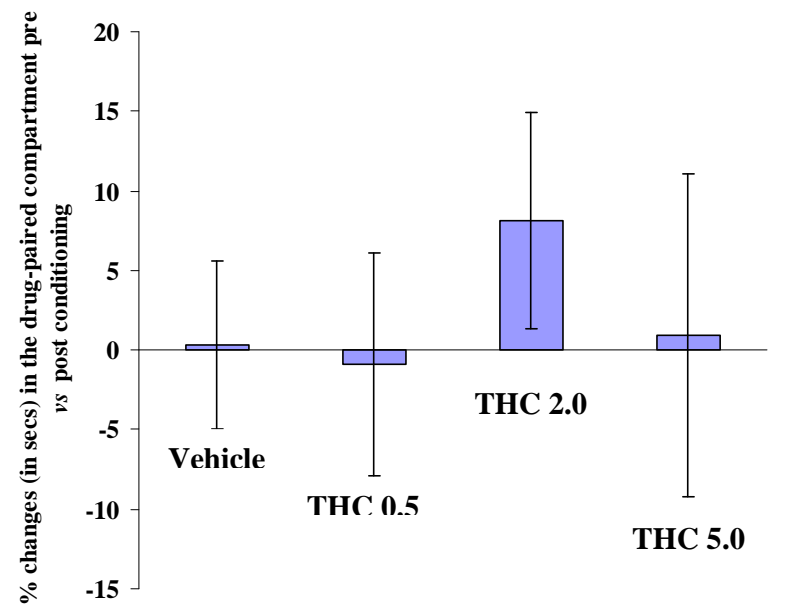

Fig. (1). Effects of treatment with vehicle $(1.0 \mathrm{ml} / \mathrm{kg}$ i.p) or THC $(0.5,2.0$ and $5.0 \mathrm{mg} / \mathrm{kg}$ i.p) given every $48 \mathrm{hrs}$ for 21 days on conditioned place preference. The data represent the percentage change (secs) spent in the drug-paired compartment during pre and post conditioning. Data represent mean \pm SEM ( $n=8$ per group). A oneway ANOVA followed by a post-hoc Tukey's multiple comparison test $p>0.05$ for comparison between group of treatment. 

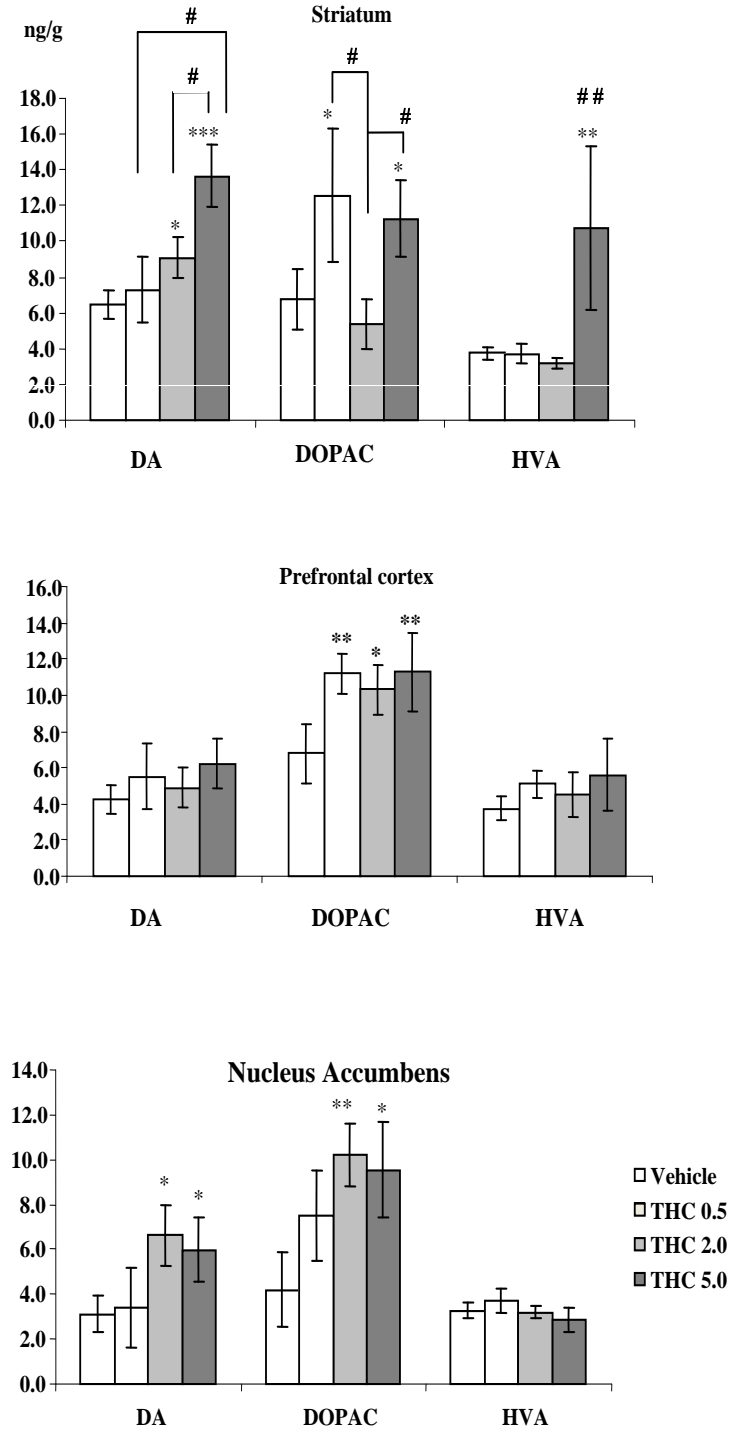

Fig. (2). Levels of dopamine and its metabolites (DOPAC and HVA) in the striatum, prefrontal cortex and nucleus accumbens after chronic administration of THC $(0.5,2.0$ and $5.0 \mathrm{mg} / \mathrm{kg}) \mathrm{com}-$ pared with vehicle treated rats. In the striatum THC at $0.5 \mathrm{mg} / \mathrm{kg}$ increased the levels of DOPAC without altering the levels of DA and HVA. In contrast, $\triangle^{9}$-THC at $2.0 \mathrm{mg} / \mathrm{kg}$ elevated the levels of DA but not DOPAC and HVA. At higher dose, $5.0 \mathrm{mg} / \mathrm{kg}$ of THC increased the levels of DA, DOPAC and HVA. In the pre-frontal cortex THC at all doses increased DOPAC but had no effect on dopamine or HVA while in the nucleus accumbens THC at 2.0 and $5.0 \mathrm{mg} / \mathrm{kg}$ increased dopamine and DOPAC but not HVA Data represent mean \pm SEM ( $\mathrm{n}=7-8$ per group). ${ }^{*} \mathrm{p}<0.05$ or $* * \mathrm{p}<0.01$ or $* * * \mathrm{p}<0.001$ vs. vehicle and ${ }^{\#} \mathrm{p}<0.05$ or ${ }^{\# \#} \mathrm{p}<0.01$ for comparison between $\triangle^{9}$-THC doses, using one-way ANOVA followed by a post-hoc Tukey's multiple comparison test.

significantly increased. In the prefrontal cortex THC at all doses $(0.5,2.0$ and $5.0 \mathrm{mg} / \mathrm{kg})$ significantly increased DOPAC ( $<<0.01, p<0.05$ and $\mathrm{p}<0.01$ respectively) but not DA or HVA. In the nucleus accumbens THC (2.0 and $5.0 \mathrm{mg} / \mathrm{kg})$ increased DA $(\mathrm{p}<0.05)$ and DOPAC $(\mathrm{p}<0.01)$ but not HVA Dopamine turnover was determined by calculating the ratio of DOPAC+HVA to DA and analysed using a oneway ANOVA followed by a post-hoc Tukey's multiple comparison test with main factors of treatment $\mathrm{x}$ region. The ratio was increased in the striatum by THC $(0.5$ and 5.0 $\mathrm{mg} / \mathrm{kg}, \mathrm{p}<0.05$ and $\mathrm{p}<0.001$ respectively) in the prefrontal cortex by THC $(2.0 \mathrm{mg} / \mathrm{kg}, \mathrm{p}<0.05)$ and in the nucleus accumbens, only THC at lower dose $(0.5 \mathrm{mg} / \mathrm{kg})$ significantly increased the ratio $(\mathrm{p}<0.05)$. The results from the measurement of 5-HT and its major metabolite 5-HIAA in the striatum and prefrontal cortex are shown in Fig. (3). These data were analysed using one-way ANOVA followed by a posthoc Tukey's multiple comparison test with dependant variables of neurochemical $\mathrm{x}$ treatment. In the striatum, THC at all doses $(0.5,2.0$ and $5.0 \mathrm{mg} / \mathrm{kg})$ significantly increased serotonin levels $(\mathrm{p}<0.05, \mathrm{p}<0.01$ and $\mathrm{p}<0.01$ respectively). However, only THC at $5.0 \mathrm{mg} / \mathrm{kg}$ increased the serotonin metabolite 5HIAA $(p<0.05)$ (Fig. 3) [10]. In the prefrontal cortex, THC had no effect at any dose on serotonin levels while THC $(2.0$ and $5.0 \mathrm{mg} / \mathrm{kg})$ significantly increased the serotonin metabolite 5HIAA $(\mathrm{p}<0.01$ and $\mathrm{p}<0.05$ respectively). There were no changes the levels of 5-HT and 5HIAA in the nucleus accumbens (data not shown). Serotonin turnover was determined by calculating the ratio of 5-HIAA to 5-HT and the analysed using one-way ANOVA followed by a post-hoc Tukey's multiple comparison test with main factors of treatment $x$ region. In the striatum, THC $(0.5$ and $2.0 \mathrm{mg} / \mathrm{kg}$ ) significantly reduced the 5HIAA:5-HT ratio $(\mathrm{p}<0.05)$. In contrast, THC $(2.0$ and $5.0 \mathrm{mg} / \mathrm{kg})$ significantly increased the 5HIAA:5-HT ratio in the prefrontal cortex $(p<0.01$ and $p<0.05$ respectively). There were no changes in serotonin turnover in the nucleus accumbens (data not shown).

\section{DISCUSSION}

\section{Conditioned Place Preference}

The conditioned place preference procedure is a classical conditioning procedure that provides an indication of drugrelated reward aversion effects in animals. It is relatively easy, quick and economical. However, it is difficult to produce a fully unbiased place conditioning protocol. The natural exploratory behaviour of rats towards colours and pattern of the walls as well as flooring texture may contribute to false positives or negatives. The use of a black and white CPP apparatus remains in common use in many laboratories and is a standard feature of many commercially available automated place conditioning systems. This study was designated into three phases, THC with $0.5,2.0$ and $5.0 \mathrm{mg} / \mathrm{kg}$ and vehicle $(1.0 \mathrm{ml} / \mathrm{kg}$ i.p, Cremophor/ethanol/saline, 1:1:18) were administered before the start of the CPP protocol. Chronic THC treatment failed to produce either place preference or place aversion. The abuse potential of cannabis in humans has been well-established; however, information about the potential rewarding effects of chronic THC is still limited. In the present study, a conditioning paradigm was used to detect drug-related reward (CPP) or aversive effects (CPA) in the rat. Chronic THC $(0.5,2.0$ and $5.0 \mathrm{mg} / \mathrm{kg})$ however failed to induce place preference at any dose. Results from previous animal studies suggest that cannabis is not a typical drug of abuse in that is does not always produce reward related responses. Indeed, under most conditions, cannabinoids fail to support self-administration $[10,11]$ or to 


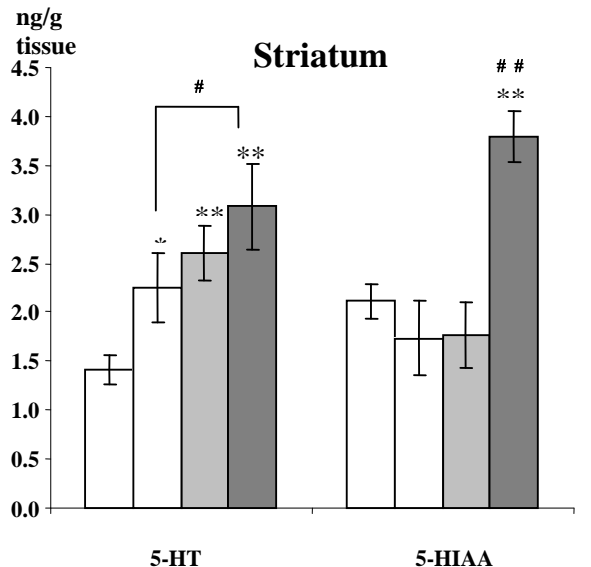

Prefrontal cortex

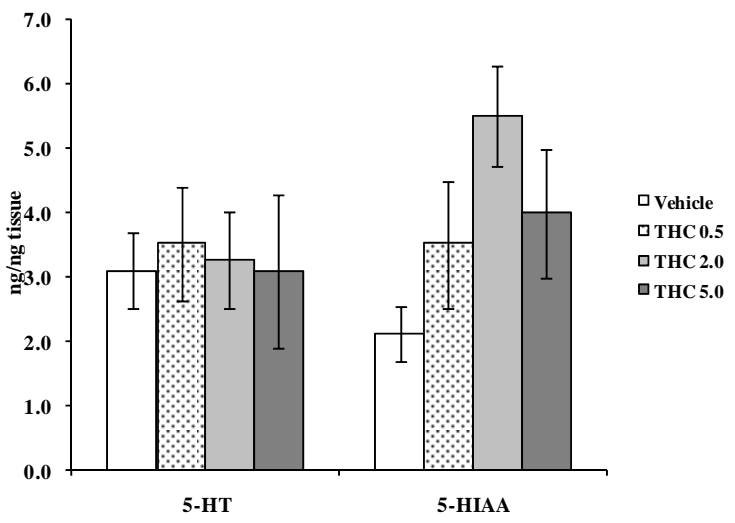

Fig. (3). Levels of serotonin and its metabolite (5HIAA) in the striatum and prefrontal cortex after chronic administration of THC $(0.5,2.0$ and $5.0 \mathrm{mg} / \mathrm{kg})$ compared with vehicle treated rats. THC at all doses significantly increased serotonin levels in striatum. However, only THC at $5.0 \mathrm{mg} / \mathrm{kg}$ increased serotonin metabolite 5HIAA levels while in the pre-frontal cortex 5HIAA levels were increased by THC 2.0 and $5.0 \mathrm{mg} / \mathrm{kg}$. Data represent mean \pm SEM $(\mathrm{n}=7-8$ per group). ${ }^{*} \mathrm{p}<0.05$ or $* * \mathrm{p}<0.001$ vs. vehicle and ${ }^{\#} \mathrm{p}<0.05$ or \# \# $\mathrm{p}<0.01$ for comparison between $\triangle^{9}$-THC doses, using one-way ANOVA followed by a post-hoc Tukey's multiple comparison test.

induce intracranial self-stimulation [12]. However, Braida and co-workers $[13,14]$ reported that sub-chronic THC and the $\mathrm{CB} 1$ agonist $\mathrm{CP} 55,940$ are able to induce conditioned place preference and support self-administration behaviour in rats. Nevertheless information regarding the effects of sub-chronic THC on reward is limited due to the frequency of false positives and differences in methodological details between laboratories; thus there are many inconsistent results from various published studies $[1,2,5-7]$. The ability of acute THC to produce positive CPP in rats [13] and mice [1] depends on the timing of injections and doses. Pre-exposure of THC $(1.0 \mathrm{mg} / \mathrm{kg})$ before the standard CPP protocol allowed subsequent THC administration to induce CPP [1]. The pre-exposure injection may eliminate the dysphoric ef- fects of the first administration of THC as the initial dysphoric effects could mask the development of positive CPP $[15,16]$. Sub-chronic treatment with the synthetic CB1 receptor agonist CP 55,940 $(20 \mu \mathrm{g} / \mathrm{kg})$ is also able to induce dose dependant CPP [17]. However, when the time interval between two injections was reduced from 48 hours to 24 hours, CP 55,940 eventually suppressed place preference [5]. THC $(1.0 \mathrm{mg} / \mathrm{kg})$ induced a positive CPP under different methodological conditions in which there was a longer wash-out period (72 hours) between drug injections [18]. However, using standard place preference conditioning protocols, with similar dose ranges, THC and synthetic cannabinoids often rather than induce CPP in rats and mice [5-7, 19-22]. These conflicting findings indicate that increasing the time interval between THC injections might qualitatively change the behavioural effects of THC because of post drug dysphoric rebound $[12,13]$. Moreover, THC is highly lipophilic with a long half life (between 64 to 94 hours) thus leading to a high accumulation of THC by the end of chronic treatment which might qualitatively change the effects of the THC in the behavioural test. These issues may explain why chronic THC fails to induce CPP using a standard place preference conditioning procedures. The issue of cannabinoid receptor desensitization should also be considered. Sim et al., [23] showed that treatment of rats with THC $(10 \mathrm{mg} / \mathrm{kg})$ for 21 days produced a marked uncoupling of $\mathrm{CB}$ receptors in most brain areas. Thus, reward behaviour initiated by acute THC administration acting via $\mathrm{CB} 1$ receptors might be unable to be reproduced after chronic drug exposure due to receptor desensitization.

\section{Measurement of Monoamine levels}

The mesolimbic DA system is part of a brain reward circuit that plays an important role in mediating the reinforcing or rewarding effects of drugs of abuse [9]. THC increases DA activity in this brain reward circuit suggesting positive reinforcing effects of THC can be accounted for by activation of DA transmission in this pathway. The present data show that the higher dose of THC significantly increased DA and its metabolites DOPAC and HVA in the striatum and NAcc. Striatal $5 \mathrm{HT}$ was also significantly increased in a dose-dependant manner. The neurochemical results are in agreement with previous studies using rat forebrain or whole brain homogenates [24] reporting increased levels of DA and $5 \mathrm{HT}$ following THC administration. THC has been shown to increase extracellular DA in the NAcc [24] and the mPFC [25], and increases DA cell firing in the VTA. In addition, it has been reported that the synthetic CB1 receptor agonists, WIN 55212-2 and CPP55,940 dose dependently and selectively increased DA and 5HT levels [2, 26-28]. Since cannabinoid receptors are not localized on DA neurons $[29,30]$, it is likely that their effect on DA release is indirectly mediated by a reduction of the release of GABA in the VTA that tonically inhibits the DA-containing neurons in the VTA. THC may activate presynaptic inhibitory CB1 receptors on the terminals of GABA interneurons, thereby reducing the release of GABA. Alternatively, THC may activate presynaptic inhibitory CB1 receptors on glutamatergic afferents to the GABA-containing neurons. These effects are mediated by $\mathrm{CB} 1$ receptors, because they are prevented by pretreatment with the CB1 antagonist, rimonabant, [16,31]. 
Therefore, the activation and increase of DA release in the VTA and the striatum is probably through disinhibition of GABA $[7,17]$. Overall, the results in the present study indicate an increase in NAcc, prefrontal cortex and striatal DA function following chronic THC but no place preference. One reason why reward could be suppressed in the presence of increased activity of reward circuits is cognitive impairment. Reward can initiate learning processes to consolidate the rewarding goal [32] and learning cues that predict its availability $[33,34]$ suggesting that the cognitive impairment caused by chronic THC exposure may interfere with learning processes and subsequently suppress reward behaviour. The CPP model assumes that rats spend more time in a location in which they passively received euphoric drugs than in an equally accessible location in which they received vehicle injection. This can be translated as positive reinforcement involving an increase over time in the frequency of behaviours that lead to a reward. Consequently, humans and animals rapidly learn cues and contexts that predict the availability of these euphoric drugs. Once learned, these cues motivate drug seeking behaviour in humans and animal models $[33,34]$. Therefore, impairment in cognitive function caused by THC exposure may suppress learned motivational cues needed for the moment-to-moment monitoring, processing, and maintenance of information. Specific impairment of memory and executive function has been identified in human cannabis users $[34,35]$.

\section{CONCLUSION}

In conclusion, chronic THC failed to induce place preference at any dose. The reasons for these dose related difference are not clear but may involve drug clearance, and aversive or cognitive effects of THC at higher dose. In addition, the present data show a marked increase in dopamine content in the striatum of rats chronically treated with THC, with parallel increases in the dopamine metabolite DOPAC, indicating increased dopaminergic activity in this region. There was also a marked increase in serotonin turnover in the prefrontal cortex of rats chronically treated with THC indicating increased serotoninergic activity in this region. In contrast serotonergic turnover in the striatum was decreased.

\section{CONFLICT OF INTEREST}

The authors confirm that this article content has no conflicts of interest.

\section{ACKNOWLEDGEMENTS}

Authors are grateful to the Universiti Putra Malaysia and Ministry of Higher Education for their financial assistances throughout the research (04-01-10-0912RU, FRGS 04-1108-626FR).

\section{ABBREVIATIONS}

$\begin{array}{lll}\mathrm{THC} & =\text { tetrahydrocannabinol } \\ \mathrm{CPP} & = & \text { conditioned place preference } \\ \mathrm{CNS} & = & \text { central nervous system } \\ \mathrm{DA} & = & \text { dopamine }\end{array}$

DOPAC $=$ 4-dihydroxyphenylacetic acid

5 -HT $=5$-hydroxytryptamine

5-HIAA $=\quad$ 5-hydroxyindoleacteic acid.

\section{REFERENCES}

[1] Valjent E, Maldonado R. A behavioural model to reveal place preference to $\triangle^{9}$-tetrahydrocannabinol in mice. Psychopharmacology $2000 ; 147: 436-8$.

[2] Tanda G, Goldberg SR. Cannabinoids: reward, dependence, and underlying neurochemical mechanisms - a review of recent preclinical data. Psychopharmacology 2003; 169: 115-34.

[3] Tzschentke TM. Measuring reward with the conditioned place preference paradigm: a comprehensive review of drug effects, recent progress and new issues. Prog Neurobiol 1998; 56: 613-72.

[4] Bevins RA, Bardo MT. Morphine-conditioned changes in locomotor activity: role of the conditioned stimulus. Exp Clin Psychopharmacol 1998; 6: 131-8.

[5] Mcgregor IS, Issakidis CN, Prior G. Aversive effects of the synthetic cannabinoid CP 55,940 in rats. Pharmacol Biochem Behav 1996; 53: 657-64.

[6] Sanudo-Pena MC, Tsou K, Delay ER, Hohman AG, Force M, Walker JM. Endogenous cannabinoids as an aversive or counterrewarding system in the rat. Neurosci Lett 1997; 223: 125-8.

[7] Cheer JF, Kendall DA, Marsden CA. Cannabinoid receptors and reward in the rat: a conditioned place preference study. Psychopharmacology 2000; 151: 25-30.

[8] Pierce RC, kumaresan V. The mesolimbic dopamine system: the final common pathway for the reinforcing effect of drugs of abuse? Neurosci Biobehac Rev 2006; 30(2): 215-38.

[9] Di chiara G, Tanda G, Bassareo V, et al. Drug addiction as a disorder of associative learning. Role of nucleus accumbens shell/extended amygdala dopamine. Ann N Y Acad Sci 1999; 877: 461-85.

[10] Leite JR, Carlini EA. Failure to obtain "cannabis-directed behavior" and abstinence syndrome in rats chronically treated with cannabis sativa extracts. Psychopharmacologia 1974; 36: 133-45.

[11] Mansbach RS, Nicholson KL, Martin BR, Balster RL. Failure of Delta(9)-tetrahydrocannabinol and CP 55,940 to maintain intravenous self-administration under a fixed-interval schedule in rhesus monkeys. Behav Pharmacol 1994; 5: 219-25.

[12] Vlachou S, Nomikos GG, Panagis G. Effects of endocannabinoid neurotransmission modulators on brain stimulation reward. Psychopharmacology 2006; 188: 293-305.

[13] Braida D, Iosue S, Pegorini S, Sala M. $\Delta$ 9-tetrahydrocannabi-nolinduced conditioned place preference and intracerebroventricular self-administration in rats. Eur J Pharmacol 2004; 506: 63-9.

[14] Braida D, Pozzi M, Cavallini R, Sala M. Conditioned place preference induced by the cannabinoid agonist CP 55,940: interaction with the opioid system. Neuroscience 2001; 104: 923-6.

[15] Lepore M, Vorel SR, Lowinson J, Gardner EL. Conditioned place preference induced by delta 9-tetrahydrocannabinol: comparison with cocaine, morphine, and food reward. Life Sci 1995; 56: 2073 80 .

[16] Ghozland S, Matthes HW, Simonin F, Filliol D, Kieffer BL, Maldonado R. Motivational effects of cannabinoids are mediated by mu-opioid and kappa-opioid receptors. J Neurosci 2002; 22: 1146-54.

[17] Tanda G, Pontieri FE, Di Chiara G. Cannabinoid and heroin activation of mesolimbic dopamine transmission by a common mul opioid receptor mechanism. Science 1997; 276: 2048-50.

[18] Robbe D, Alonso G, Duchamp F, Bockaert J, Manzoni OJ. Localization and mechanisms of action of cannabinoid receptors at the glutamatergic synapses of the mouse nucleus accumbens. J Neurosci 2001; 21: 109-16.

Mallet PE, Beninger RJ. The cannabinoid CB1 receptor antagonist RIMONABANT attenuates the memory impairment produced by delta9-tetrahydrocannabinol or anandamide. Psychopharmacology 1998; 140: 11-9.

Mallet PE, Beninger RJ. $\Delta$ 9-tetrahydrocannabinol, but not the endogenous cannabinoid receptor ligand anandamide, produces conditioned place avoidance. Life Sci 1998; 62(26): 2431-9.

Chaperon F, Soubrie P, Puech AJ, Thiebot MH. Involvement of central cannabinoid (CB1) receptors in the establishment of place conditioning in rats. Psychopharmacology 1998; 135: 324-32. 
[22] Zimmer A, Yaljent E, Konig M. et al. Absence of delta -9tetrahydrocannabi-nol dysphoric effects in dynorphin-deficient mice. J Neurosci 2001; 21: 9499-505.

[23] Sim LJ, Hampson RE, Deadwyler SA, Childers SR. Effects of chronic treatment with $\Delta$ 9-tetrahydrocannabinol on cannabinoidstimulated [35S]GTPgammaS autoradiography in rat brain. J Neurosci. 1996;16:8057-66.

[24] Sofia RD, Ertel RJ, Dixit BN, Barry H. The effect of 1tetrahydrocannabinol on the uptake of serotonin by rat brain homogenates. Eur J Pharmacol 1971; 6(2): 257-9.

[25] Chen J, Paredes W, Lowinson JH, Gardner EL. $\Delta$ 9-tetrahydrocannabinol enhances presynaptic dopamine efflux in medial prefrontal cortex. Eur J Pharmacol 1990; 190: 259-62.

[26] Chen J, Paredes W, Li J, Smith D, Lowinson J, Gardner EL. $\Delta$ 9tetrahydrocannabinol produces naloxone- blockable enhancement of presynaptic basal dopamine efflux in nucleus accumbens of conscious, freely- moving rats as measured by intracerebral microdialysis. Psychopharmacology 1990; 102: 156-62.

[27] Malone DT, Taylor DA. Modulation by fluoxetine of striatal dopamine release following Delta9-tetrahydrocannabinol: a microdialysis study in conscious rats. Br J Pharmacol 1999; 128: 21-6.

[28] Melis M, Gessa GL, Diana M. Different mechanisms for dopaminergic excitation induced by opiates and cannabinoids in the rat midbrain. Prog Neuropsychopharmacol Biol Psychiatry 2000; 24 : 993-1006.

[29] Arevalo C, De Miguel R, Hernandez-Tristan R. Cannabinoid effects on anxiety-related behaviours and hypothalamic neurotransmitters. Pharmacol Biochem Behav 2001; 70: 123-31.

[30] Herkenham M, Lynn AB, Johnson MR, Melvin LS, De Costa BR, Rice KC. Characterization and localization of cannabinoid receptors in rat brain: a quantitative in vitro autoradiographic study. $\mathrm{J}$ Neurosci 1991; 11: 563-83.

[31] Szabo B, Muller T, Koch H. Effects of cannabinoids on dopamine release in the corpus striatum and the nucleus accumbens in vitro. $J$ Neurochem 1999; 73: 1084-9.

[32] Repovs G, Baddeley A. The multi-component model of working memory: explorations in experimental cognitive psychology. Neuroscience 2006; 139: 5-21.

[33] Domjan M. Stepping outside the box in considering the $\mathrm{C} / \mathrm{T}$ ratio. Behav Processes 2003; 62: 103-14.

[34] Block RI. Does heavy marijuana use impair human cognition and brain function? JAMA 1996; 275: 560-1.

[35] Block RI, Ghoneim MM. Effects of chronic marijuana use on human cognition. Psychopharmacology 1993; 110: 219-28.

Received: February 16, 2012

Revised: April 20, 2012

Accepted: April 25, 2012

(C) Moklas et al.; Licensee Bentham Open.

This is an open access article licensed under the terms of the Creative Commons Attribution Non-Commercial License (http://creativecommons.org/licenses/by-nc/3.0/) which permits unrestricted, non-commercial use, distribution and reproduction in any medium, provided the work is properly cited. 